\title{
Coupling Metallic Nanostructures to Thermally Responsive Polymers Allows the Development of Intelligent Responsive Membranes
}

\author{
J. Rubén Morones-Ramírez \\ Facultad de Ciencias Químicas, Universidad Autónoma de Nuevo León, Pedro de Alba S/N, San Nicolás de Los Garza, NL, Mexico \\ Correspondence should be addressed to J. Rubén Morones-Ramírez; morones.ruben@gmail.com
}

Received 5 December 2013; Accepted 7 May 2014; Published 22 May 2014

Academic Editor: Iseult Lynch

Copyright (C) 2014 J. Rubén Morones-Ramírez. This is an open access article distributed under the Creative Commons Attribution License, which permits unrestricted use, distribution, and reproduction in any medium, provided the original work is properly cited.

\begin{abstract}
Development of porous membranes capable of controlling flow or changing their permeability to specific chemical entities, in response to small changes in environmental stimuli, is an area of appealing research, since these membranes present a wide variety of applications. The synthesis of these membranes has been mainly approached through grafting of environmentally responsive polymers to the surface walls of polymeric porous membranes. This synergizes the chemical stability and mechanical strength of the polymer membrane with the fast response times of the bonded polymer chains. Therefore, different composite membranes capable of changing their effective pore size with environmental triggers have been developed. A recent interest has been the development of porous membranes responsive to light, since these can achieve rapid, remote, noninvasive, and localized flow control. This work describes the synthesis pathway to construct intelligent optothermally responsive membranes. The method followed involved the grafting of optothermally responsive polymer-metal nanoparticle nanocomposites to polycarbonate tracketched porous membranes (PCTEPMs). The nanoparticles coupled to the polymer grafts serve as the optothermal energy converters to achieve optical switching of the pores. The results of the paper show that grafting of the polymer and in situ synthesis of the metallic particles can be easily achieved. In addition, the composite membranes allow fast and reversible switching of the pores using both light and heat permitting control of fluid flow.
\end{abstract}

\section{Introduction}

Porous membranes capable of controlling flow or changing their permeability to specific chemical entities, in response to small changes in environmental stimuli, are an area of appealing research, since these membranes present potential applications as sensors, in bioseparations, as drug delivery systems, and as valves that serve to interconnect microfluidic systems and control interflow $[1,2]$. The synthesis of these environmentally responsive membranes has been mainly approached through grafting of environmentally responsive polymers to the surface walls of polymeric porous membranes. This synergizes the chemical stability and mechanical strength of the polymer membrane with the fast response times of the chemically bonded free-ended polymer chains. Therefore, different composite membranes capable of changing their effective pore size with environmental triggers such as $\mathrm{pH}$ and ionic strength [3, 4], temperature [5], and chemical moieties, such as glucose [6], have been developed. However, a recent interest has been the development of porous membranes responsive to light, since these present advantages potentially achieve rapid, remote, noninvasive, and localized flow control [7-10]. This work describes the development of a synthesis pathway to achieve grafting of optothermally responsive polymer-metal nanoparticle nanocomposites to polycarbonate track-etched porous membranes (PCTEPMs) to build an optothermally responsive porous membrane.

\section{Results and Discussion}

2.1. Synthesis of Thermally Responsive Membranes. Different methods have been reported in the literature to achieve 
grafting of polymer chains to the surface of porous membranes and these techniques fall predominantly into two categories: "grafting to" and "grafting from." We found in the literature that growing polymers from the surface, "grafting from," rather than functionalizing the polymers, "grafting to," allows better control on reaction and permits obtaining higher polymer densities [11]. Several surface "grafting from" techniques have been reported in the literature: chemical grafting [11], plasma [12, 13], radiation $[3,9,14,15]$, and photoinduced grafting $[16,17]$. Most of these techniques presented disadvantages for our systems. For example, with the use of photoinduced grafting, it is difficult to achieve grafts in the membrane pores; chemical grafting requires handling of poisonous initiators; and finally, using radiation requires expensive machinery and the bulk properties of the membranes are affected. However, the plasma-induced surface polymerization technique offered us a great alternative since it has been shown to be able to perform grafting of poly $\mathrm{N}$-isopropyl acrylamide (PNIPAM) on both the inside of the pores and on the surface of the membrane, additionally it maintains the bulk properties of the polycarbonate tracketched porous membrane PCTEPM unchanged [12].

For the grafting of PNIPAM to the PCTEPM we employed a plasma-induced technique that has been previously reported in the literature [13] and, which, consists of exposing the PCTEPM to an oxygen (air is used for our case) plasma cloud that creates metastable chemical radicals on the surface of the membrane. When the treated membranes are exposed to a monomer solution, the surface polymerization is initiated without the addition of an initiator, and so the polymer is grafted to the membrane surface at the top of the membrane and the walls of the pores but not at the bottom where the plasma cloud could not reach.

The plasma-induced grafting presents a major advantage for our synthesis since it can achieve a control over the location of the grafting of the PNIPAM chains, either on the membrane surface or to the walls of the pores [13]; additionally, the plasma treatment only affects the outer properties of the membrane, maintaining the bulk properties of the membrane unchanged [12]. The most important mechanism in the control of the grafting location of the PNIPAM, either in the surface or inside the pores, is the capability of the monomers to diffuse into the pore and form chains. Therefore, reactions that do not promote monomer diffusion, such as low temperature environments or using solvents that do not wet pores efficiently, promote preferential surface grafting $[4,5,18]$. Another factor is that diffusion of the monomers into the pores should occur initially in the polymerization, since, as the grafts begin to grow on the surface, they cover the pores and steric effects present a barrier for monomer diffusion to reach the pores [16].

We controlled the grafting location of the PNIPAM by wetting the membrane using ethanol prior to the initiation of the surface polymerization. It has been shown previously that ethanol allows a more efficient wetting of the PCTEPM and so promotes grafting of the polymer in the pores [4]. In addition, we ran the polymerization at $30^{\circ} \mathrm{C}$, which is just below the lower critical solution temperature (LCST), and increased grafting efficiency. Localization of the polymer grafts on our membrane samples was analyzed using SEM. Figures 1(a)1(b) show the surface and interior pore structure, respectively, of the PCTEPM control. Surface of the PNIPAM grafted membrane is shown in Figure 1(c) and the interior structure of the grafted pores can be observed in Figure 1(d). The physical differences observed in the scanning electron microscope (SEM) images between the control PCTEPMs and the grafted polycarbonate track-etched porous membranes (gPCTEPMs) are very slight, since both the PNIPAM grafts and the polycarbonate, of which the PCTEPMs are made of, are amorphous polymers. Nonetheless, in the grafted membranes the pores seem to have a thin film covering them. In addition, other slight differences such as a loss of sharpness at the edges can be appreciated between the samples (Figures $1(\mathrm{a})-1(\mathrm{~d}))$.

2.2. Synthesis of Optothermally Responsive Membranes. The coupling of metal particles to the PNIPAM grafts in the PCTEPM is approached through an in situ reduction of a salt of silver. The membrane with the PNIPAM grafts is submerged in an aqueous solution of the metallic salt, followed by the addition of AsA (ascorbic acid), the reducing agent. After the addition of the reducing agent, the aqueous silver solution follows a dramatic change due to the reduction of silver, where the aqueous silver solution reacts immediately to form large aggregates in the surface walls, which give an apparent mirror like surface. However, when the membranes are extracted from the solution they present very light colors, due to the SPR (surface plasmon resonance) exhibited by nanoparticles with a characteristic yellowish color. This proves that the PNIPAM grafted in the porous membranes is acting as a stabilizing and capping agent in the synthesis of the metallic nanoparticles, as predicted by our previous work that describes PNIPAM as a capping agent for metallic nanoparticle formation $[19,20]$.

The localization of the polymer grafts on each PCTEPM sample and the size and shape of the synthesized nanoparticles were analyzed using SEM. As a control of the in situ reduction of the silver metallic salt, Figure 2(a) shows the silver nanoparticles formed on the PCTEPM without PNIPAM grafts. Most of the particles are aggregated and are very inhomogeneously dispersed in the membrane. However, we can further observe in Figures 2(b)-2(c) that silver nanoparticles synthesized in situ at the surface of the membrane show spherical morphologies and nanoparticles that are dispersed all throughout the membrane surface. We have, therefore, proven that the PNIPAM grafts coupled with metallic nanoparticles are capable of controlling the synthesis of silver nanoparticles. Additionally, Figure 2(d) shows the presence of particles in PCTEPM pores, which is also an indirect proof of the presence of PNIPAM grafts within the pores of the porous membranes for our surface grafting polymerization synthesis.

From Figures 3(a) and 3(b) it can be observed that the particles formed within the pores present a $30 \%$ increase in average size when compared to the particles formed on the surface of the membrane. Additionally, the size distribution for the particles formed inside the pores (stdev $16 \mathrm{~nm}$ ) has 


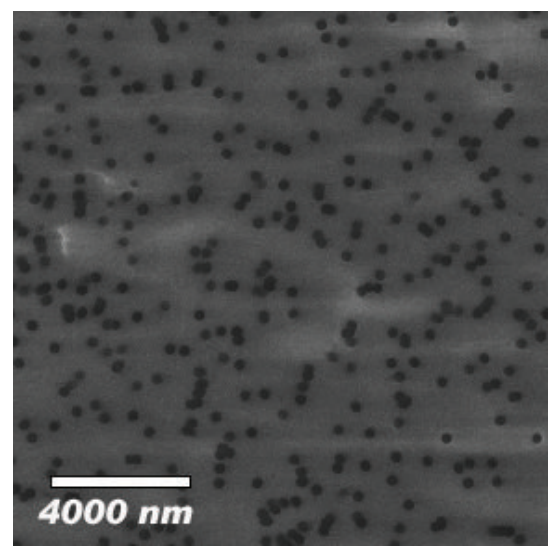

(a)

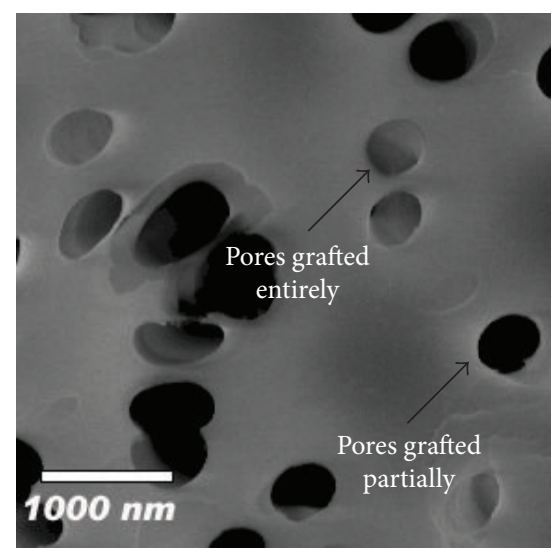

(c)

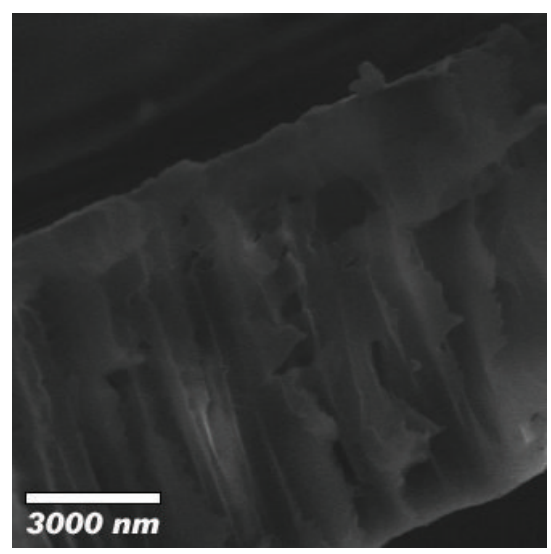

(b)

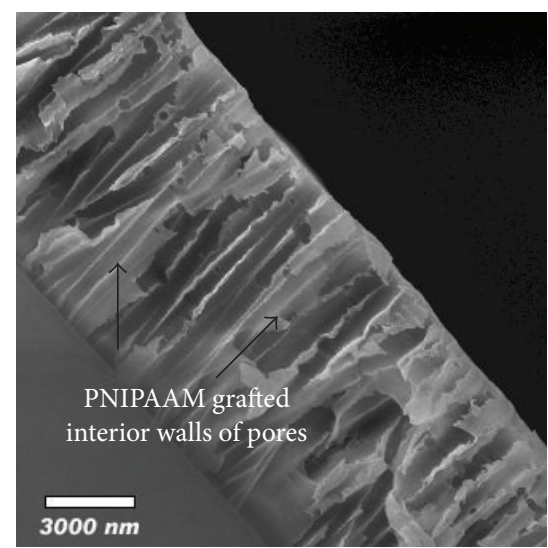

(d)

FIGURE 1: Show scanning electron microscopy micrographs of the polycarbonate porous membranes. (a) Control PCTEPM surface without PNIPAM grafts. (b) Clean cut internal structure of the control PCTEPM without PNIPAM grafts. (c) Surface and (d) internal structure of PCTEPM that has been grafted with PNIPAM.

$45 \%$ better monodispersity than that of the particles formed in the surface (stdev $34 \mathrm{~nm}$ ); we attribute this to a diffusion effect within the nanopores that aids in a controlled synthesis of the nanoparticles.

\subsection{Heat Permeability Switching Experiments. The PNIPAM} chains in an aqueous medium grafted to the surface of the PCTEPM, when exposed to temperature, are expected to act as a mechanical valve that controls flow. We tested the temperature dependent average flows through the porous membranes using gravity-driven flow with time changing hydrostatic pressure starting at $500 \mathrm{~Pa}$ and ending at $200 \mathrm{~Pa}$, since the water column decreases as the water flows. Membranes with and without silver nanoparticles were tested for three different switching cycles and produced reproducible results, meaning the polymer and nanocomposites were grafted stably to the membranes. Figure 4(a) shows the relative flow rate as a function of water temperature. It can be observed that for the grafted membranes the average flow rates are always lower than those for the ungrafted PCTEPM. We were able to achieve four fold differences in the flow rates for the membranes where the grafting was done compared to the nongrafted membranes. For the grafted membranes, we were able to achieve very low flow rates with an upper limit in the order of $1 \mu \mathrm{L} / \mathrm{s} \mathrm{cm}^{2}$ when the PNIPAM is below the LCST. This indicates that the polymer below the LCST is able to achieve nearly total blockage of the pores to convective flow.

Studies with track-etched membranes often calculate effective pore diameters based on Hagen-Poiseuille's law for the flow rate in a cylinder: $V / \Delta t=$ flowrate $=\pi \Delta \operatorname{Pr}^{4} / 8 \eta L$, where $V$ is volume of the permeate, $\Delta P$ is the transmembrane pressure, $r$ is the pore radius (assuming homogenous sized pores in the membrane), $\eta$ is the viscosity of the permeate, and $L$ is the capillary length (membrane thickness). The flow measurements here were performed with time-varying pressure and, therefore, in the Hagen-Poiseuille equation the time averaged volumetric flow rate and pressure drop are used. The time average pressure drop is constant in all flow experiments here. In order to exclude viscosity changes with temperature and to be independent of the exact time-dependence of the pressure decrease, all results are relative to the control membrane. Therefore, pore sizes are calculated using the following equation: flowrate $_{(\text {control, } T)} /$ flowrate $_{(\text {grafted }, T)}=r_{(\text {control }, T)}^{4} / r_{(\text {grafted,T) }}^{4}$. 


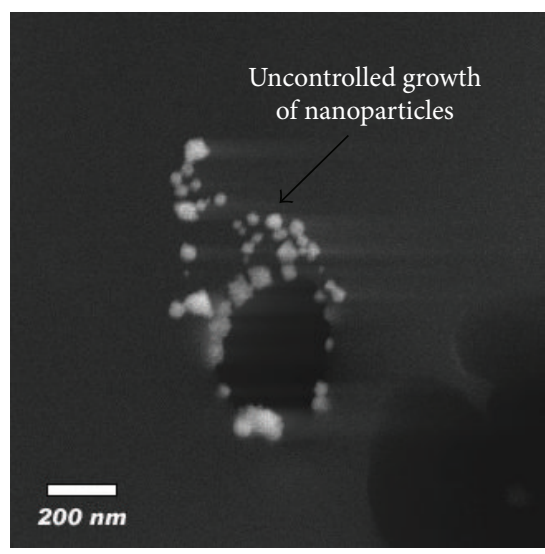

(a)

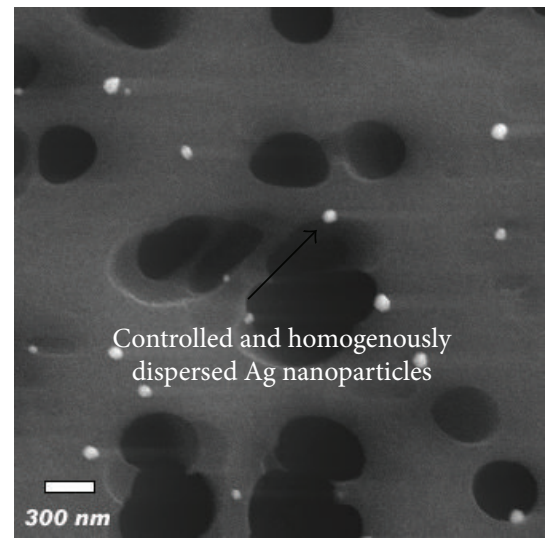

(c)

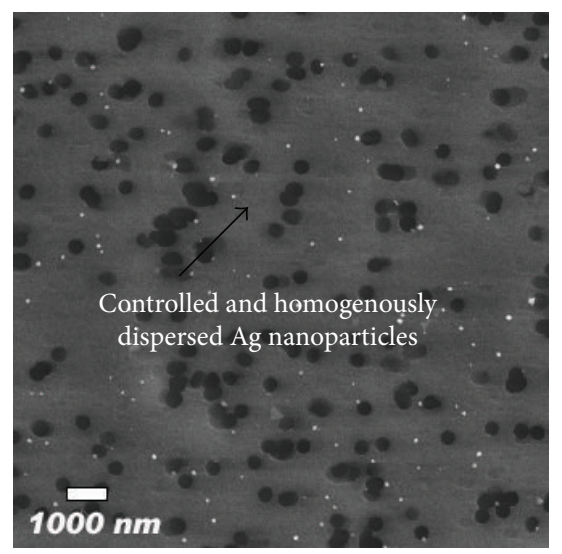

(b)

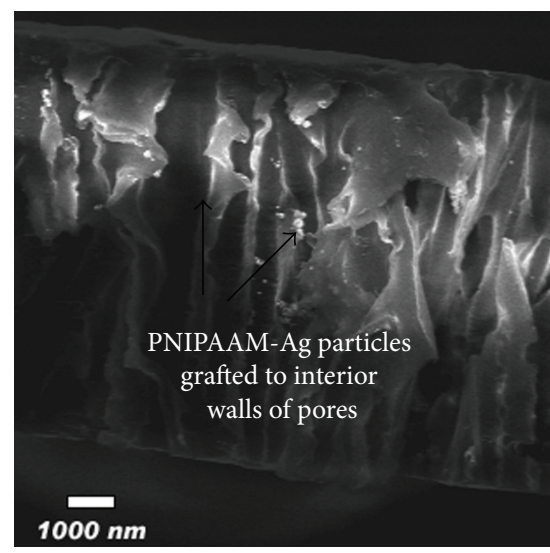

(d)

FIGURE 2: Show scanning electron microscopy micrographs of the porous polycarbonate membranes. (a) Control PCTEPM surface without PNIPAM grafts after silver nanoparticles have been synthesized in situ. (b)-(c) Surface and (d) internal structure of PCTEPM that has been grafted with PNIPAM and silver nanoparticles has been synthesized in situ.

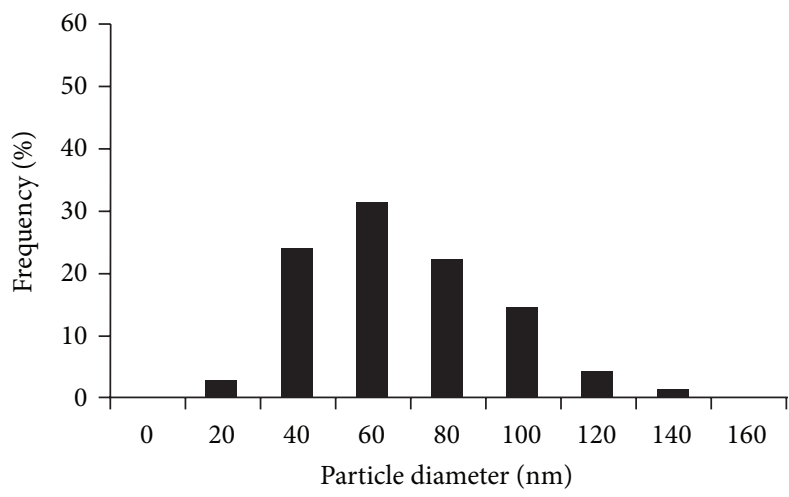

(a)

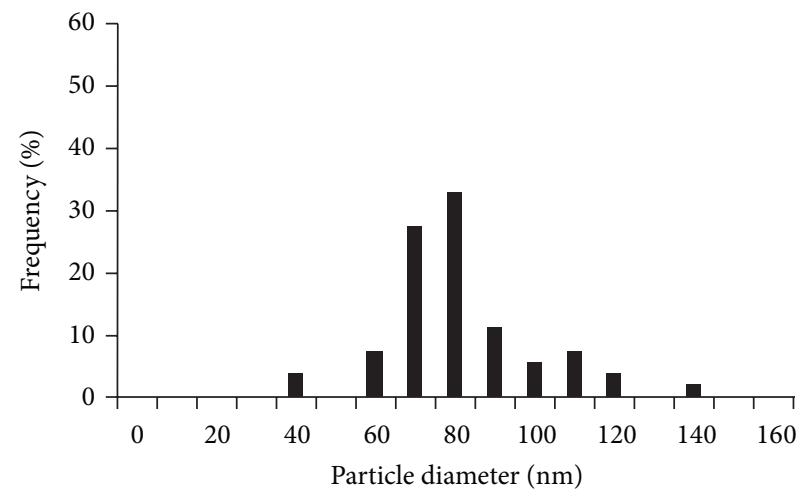

(b)

FIgURE 3: (a) Size distribution of the silver nanoparticles synthesized in situ on the surface of the gPCTEPM. (b) Size distribution of the silver nanoparticles synthesized in situ within the pores of the gPCTEPM.

Assuming simple Hagen-Poiseuille's flow, however, is inaccurate because the thickness of surface-grafted polymer layer in a cylindrical conduit is flow rate dependent. Except for very low flow rates, the polymer layer has been proposed to swell inside de pore due to shear, or flatten at the top surface of the pore if the order of the polymer brush is not very high and not all chains are pointing towards the flow (incomplete grafting) [21-23]. Additionally, if the polymer graft would be considered as a continuous gel with viscoelastic properties, which could be induced by embedding silver nanoparticles 


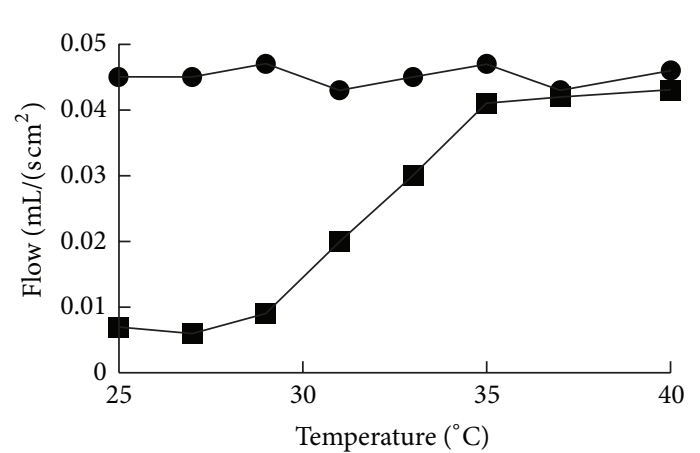

(a)

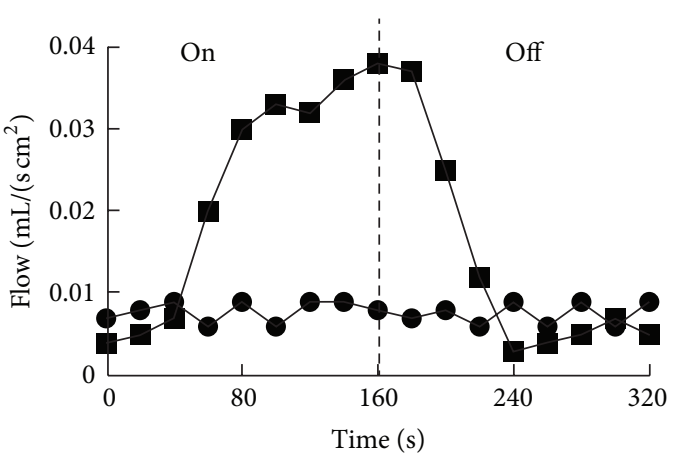

(b)

FIGURE 4: Permeability switching of gPCTEPMs in response to (a) heat and (b) light. Circles correspond to the control ungrafted membrane in (a) and grafted without nanoparticles in (b). Squares correspond to grafted membrane in (a) and grafted membrane with silver nanoparticles in (b).

into the polymers, instabilities at the interface can develop even at very low Reynolds numbers [24, 25].

Despite the shortcomings of the Hagen-Poiseuille approximation, the pore size changes observed for the membranes with grafts polymerized are shown in Figures 4(a)-4(b). The pore sizes in the membranes show an effective change in pore diameters of $\sim 70 \mathrm{~nm}$ (stdev $8 \mathrm{~nm}$ ) changing from an open pore in the collapsed state of $350 \mathrm{~nm}$ to a diameter of $280 \mathrm{~nm}$ in the hydrated state below the LCST.

The $\sim 70 \mathrm{~nm}$ (stdev $8 \mathrm{~nm}$ ) change in pore size between collapsed and swollen state of the PNIPAM chains obtained in this work is similar to pore changes reported in the literature, using the Hagen-Poiseuille's equation and using plasmainduced grafting $[3,12,17,26]$. Switching responses with bigger change in pore sizes, from 50 to $200 \mathrm{~nm}$, have been achieved using hydrogels; however, these systems present very long response times [27].

A very similar experimental setup and pore size calculations (Hagen Poiseulle) used for the thermal switching experiments were used for the light-induced permeability changes in the functionalized porous membranes (Figure 4(b)). We plotted fluid flow through the membrane as a function of time at which the membrane was exposed to a $430 \mathrm{~nm}$ laser beam. The behavior observed was very similar to that of the thermally-induced switching. This is not at all surprising since the mechanical effect should be the same, but the actuator, light, is the only difference. We were able to see that flow control was very fast and reversible. $50 \%$ of the increased total flow capability of the membrane is achieved within less than a minute of light exposure, which makes these membranes ideal for a wide range of applications.

\section{Materials and Methods}

3.1. Materials. Deionized water with a resistivity of at least $(18.0 \mathrm{M} \Omega \mathrm{cm})$ (E-Pure, Barnstead Thermolyne) was used in all experiments. N-Isopropyl acrylamide (NIPAM) (Fisher Scientific) was recrystallized once in hexane (Fisher Scientific) and stored at $-20^{\circ} \mathrm{C}$ until use. Ascorbic acid (AsA) (Fisher Scientific) and silver nitrate $\left(\mathrm{AgNO}_{3}\right)$ (Sigma
Aldrich) were used as purchased. Polycarbonate tracketched (PCTE) membranes $400 \mathrm{~nm}$ and $60 \mu \mathrm{m}$ diameter were bought from Whatman and used as purchased.

3.2. Surface Grafting of PNIPAM on Pores of PCTE Porous Membranes Using Plasma-Graft Pore Filling Surface Polymerization. Linear PNIPAM chains were grafted to both the surface and into the pores of the PCTEPM of $400 \mathrm{~nm}$ diameter by a plasma-induced grafting polymerization technique reported by Xie et al. [13]. The reaction involved placing the membranes in a transparent glass tube that is filled with air, without any flow, and then evacuated at $350 \mu$ Torr. The substrate is then treated with a steady cloud of air plasma at $30 \mathrm{~W}$ for $60 \mathrm{~s}$. Immediately after treatment, the membrane is immersed in ethanol for $2 \mathrm{~s}$ and, subsequently, the membrane is submerged in a $5 \mathrm{wt} \%$ monomer aqueous solution and left to polymerize without initiator for $2-4 \mathrm{hrs}$. The reaction was performed at both room temperature and $30^{\circ} \mathrm{C}$. Following the reaction, the membrane is well rinsed three times in deionized water and then it is left in $50 \mathrm{~mL}$ of deionized water for $24 \mathrm{hrs}$ to remove any unreacted monomer.

3.3. In Situ Synthesis of Silver Nanoparticles on PNIPAM Grafts. We achieved the growth of silver nanoparticles in the pores by two approaches: the first involved the following steps. The PCTEPM with surface-grafted PNIPAM are submerged into a $5 \mathrm{~mL}$ aqueous solution of $\mathrm{AgNO}_{3}(0.001 \mathrm{M})$ and left for $1 \mathrm{hr}$ to impregnate. Afterward AsA is added to the $5 \mathrm{~mL}$ aqueous solution to achieve a $0.003 \mathrm{M}$ solution, and the solution is stirred for 10 minutes. Finally, the membranes are rinsed with deionized water and sonicated for 10 minutes to remove any particles that are not within the PNIPAM grafts.

As a second approach, in order to drive the synthesis of silver nanoparticles inside pores of the membrane, a PNIPAM grafted membrane was taped to the end of the thicker part of a Pasteur pipette and submerged into a $5 \mathrm{~mL}$ aqueous solution of AsA ( $0.03 \mathrm{M})$ contained in a $10 \mathrm{~mL}$ volume container. Immediately afterwards an aqueous solution of $\mathrm{AgNO}_{3}(0.01 \mathrm{M})$ was poured into the cylindrical tube, making sure there was no positive or negative pressure from 
the column of the aqueous solution of $\mathrm{AgNO}_{3}$. This allowed both the AsA and $\mathrm{AgNO}_{3}$ to diffuse in opposite directions inside the membrane, due to concentration gradients, and allowed nanoparticle reduction within the pores.

A size distribution analysis of the particles at the different synthesis conditions was then carried out through the analysis of different SEM micrographs. The distribution was obtained by analyzing the size of 400 particles chosen at random. With the data on particle size, average particle size and standard deviation (stdev) were determined.

3.4. Scanning Electron Microscopy (SEM). SEM images were obtained with a LEO 1530 scanning electron microscope at an acceleration voltage of $10 \mathrm{kV}$. The specimens were prepared placing the membranes on a carbon-coated conducting tape and then the samples were coated unless specified at each image shown with silver, which allowed better quality images and higher resolution. Clean-cut images, that allowed analysis of the interior of the membrane, were obtained by breaking the membrane while it was submerged into liquid nitrogen. The membrane was soaked in water prior to putting it into liquid nitrogen.

3.5. Thermal Control of Fluid Flow. Permeability changes in the membrane induced by temperature were determined by measuring water flow through the membranes for three different switching cycles. The membrane was taped to the end of a Pasteur pipette (diameter of $5 \mathrm{~mm}$ ) containing a water column of $5 \mathrm{~cm}(\sim 1 \mathrm{~mL})$. Afterwards, $0.43 \mathrm{~mL}$ was allowed to flow through the membranes. By the setup of the experiment, the differential pressure was not kept constant, since the water column changed height as the flow passed. The respective times were recorded and the average flow rate was determined every $20 \mathrm{~s}$. The volume of water flowing through the membrane was collected in a tube and differences in height were determined every 20 seconds. Afterwards, the differences in height were converted to volumetric flow $(\mathrm{mL} / \mathrm{s})$. Additionally, the times for the sample membrane were normalized to an untreated PCTEPM for converting flow rates using the Hagen-Poiseuille equation to an effective pore size. The temperature switching experiments of the membranes were performed by heating the volume of flowing water to different temperatures between $27^{\circ} \mathrm{C}$ and $40^{\circ} \mathrm{C}$.

3.6. Optical Control of Fluid Flow. For the experiments to test water flow control in membranes using light, the same setup and procedure that were followed for testing thermal control of flow were followed. However, instead of temperature, the light switching experiments of the membranes were performed by exposing the membrane to a laser beam of light at a wavelength of $430 \mathrm{~nm}$ for 160 seconds. The flowing water was previously heated to $31^{\circ} \mathrm{C}$ and the average water flow was recorded in response to time. The water flow measurements initiated at the time the membrane was exposed to the laser beam and until the laser was turned off and the flow returned to the initial conditions.

\section{Conclusions}

We successfully developed a synthesis method to incorporate PNIPAM-metal grafts into polycarbonate porous membranes to create responsive valves. The synthesis involved initial grafting of the PNIPAM followed by reduction in situ of a metallic salt. We were able to control size and shape of the nanocomposite by using the PNIPAM grafts as capping and stabilizing agents for silver and so achieved successful coupling of both metals to PNIPAM and immobilized the composite in the membranes.

The synthesized membranes showed, experimentally, a switch in response to temperature and light and achieved differences in flow. Therefore this synthesis methodology is a step forward to the development of responsive membranes with potential applications as optically responsive valves for the spatiotemporal delivery of bioactive agents, cell array, and advanced cell culture applications, among others.

\section{Conflict of Interests}

The author declares that there is no conflict of interests regarding the publication of this paper.

\section{Acknowledgments}

This project was funded through PAICyT and PROVERICIT from the Universidad Autonoma de Nuevo Leon. This project was also supported by Paicyt, with support from the Universidad Autónoma de Nuevo León. The authors would like to thank the University for the funding.

\section{References}

[1] T.-C. Kuo, D. M. Cannon Jr., M. A. Shannon, P. W. Bohn, and J. V. Sweedler, "Hybrid three-dimensional nanofluidic/ microfluidic devices using molecular gates," Sensors and Actuators A: Physical, vol. 102, no. 3, pp. 223-233, 2003.

[2] S. Hediger, J. Fontannaz, A. Sayah, W. Hunziker, and M. A. M. Gijs, "Biosystem for the culture and characterization of epithelial cell tissues," Sensors and Actuators B: Chemical, vol. 63, no. 1, pp. 63-73, 2000.

[3] J. Hautojarvi, K. Kontturi, J. H. Näsman, B. L. Svarfvar, P. Viinikka, and M. Vuoristo, "Characterization of graft-modified porous polymer membranes," Industrial and Engineering Chemistry Research, vol. 35, no. 2, pp. 450-457, 1996.

[4] T. Peng and Y.-L. Cheng, "pH-responsive permeability of PE-g-PMAA membranes," Journal of Applied Polymer Science, vol. 76, no. 6, pp. 778-786, 2000.

[5] T. Peng and Y.-L. Cheng, "Temperature-responsive permeability of porous PNIPAAm-g-PE membranes," Journal of Applied Polymer Science, vol. 70, no. 11, pp. 2133-2142, 1998.

[6] S. Cartier, T. A. Horbett, and D. B. Ratner, "Glucose-sensitive membrane coated porous filters for control of hydraulic permeability and insulin delivery from a pressurized reservoir," Journal of Membrane Science, vol. 106, no. 1-2, pp. 17-24, 1995.

[7] S. K. Kumar and J.-D. Hong, "Photoresponsive ion gating function of an azobenzene polyelectrolyte multilayer spin-selfassembled on a nanoporous support," Langmuir, vol. 24, no. 8, pp. 4190-4193, 2008. 
[8] C. J. Brinker, "Evaporation-induced self-assembly: functional nanostructures made easy," MRS Bulletin, vol. 29, no. 9, pp. 631-640, 2004.

[9] A. Nayak, H. Liu, and G. Belfort, "An optically reversible switching membrane surface," Angewandte ChemieInternational Edition, vol. 45, no. 25, pp. 4094-4098, 2006.

[10] J. R. Morones-Ramirez, "Bioinspired synthesis of optically and thermally responsive nanoporous membranes," NPG Asia Materials, vol. 5, article e52, 2013.

[11] H. Alem, A. Duwez, P. Lussis, P. Lipnik, A. M. Jonas, and S. Demoustier-Champagne, "Microstructure and thermoresponsive behavior of poly( $\mathrm{N}$-isopropylacrylamide) brushes grafted in nanopores of track-etched membranes," Journal of Membrane Science, vol. 308, no. 1-2, pp. 75-86, 2008.

[12] J. Huang, X. Wang, X. Chen, and X. Yu, “Temperaturesensitive membranes prepared by the plasma-induced graft polymerization of $\mathrm{N}$-isopropylacrylamide into porous polyethylene membranes," Journal of Applied Polymer Science, vol. 89, no. 12, pp. 3180-3187, 2003.

[13] R. Xie, L. Chu, W. Chen, W. Xiao, H. Wang, and J. Qu, "Characterization of microstructure of poly( $\mathrm{N}$-isopropylacrylamide)grafted polycarbonate track-etched membranes prepared by plasma-graft pore-filling polymerization," Journal of Membrane Science, vol. 258, no. 1-2, pp. 157-166, 2005.

[14] N. I. Shtanko, V. Y. Kabanov, P. Y. Apel, M. Yoshida, and A. I. Vilenskii, "Preparation of permeability-controlled track membranes on the basis of "smart" polymers," Journal of Membrane Science, vol. 179, no. 1-2, pp. 155-161, 2000.

[15] M. Ulbricht, "Photograft-polymer-modified microporous membranes with environment-sensitive permeabilities," Reactive and Functional Polymers, vol. 31, no. 2, pp. 165-177, 1996.

[16] B. Yang and W. Yang, "Thermo-sensitive switching membranes regulated by pore-covering polymer brushes," Journal of Membrane Science, vol. 218, no. 1-2, pp. 247-255, 2003.

[17] W. Lequieu, N. I. Shtanko, and F. E. Du Prez, "Track etched membranes with thermo-adjustable porosity and separation properties by surface immobilization of poly $(\mathrm{N}$ vinylcaprolactam)," Journal of Membrane Science, vol. 256, no. 1-2, pp. 64-71, 2005.

[18] T. Peng and Y.-L. Cheng, "PNIPAAm and PMAA co-grafted porous PE membranes: living radical co-grafting mechanism and multi-stimuli responsive permeability," Polymer, vol. 42, no. 5, pp. 2091-2100, 2001.

[19] J. Morones and W. Frey, "Environmentally sensitive silver nanoparticles of controlled size synthesized with PNIPAM as a nucleating and capping agent," Langmuir, vol. 23, no. 15, pp. 8180-8186, 2007.

[20] J. R. Morones and W. Frey, "Room temperature synthesis of an optically and thermally responsive hybrid PNIPAM-gold nanoparticle," Journal of Nanoparticle Research, vol. 12, no. 4, pp. 1401-1414, 2010.

[21] E. M. Sevick, "Shear swelling of polymer brushes grafted onto convex and concave surfaces," Macromolecules, vol. 29, no. 21, pp. 6952-6958, 1996.

[22] G. S. Grest, Polymers in Confined Environments, vol. 138 of Advances in Polymer Science, 1999.

[23] P. Chokshi and V. Kumaran, "Stability of the flow of a viscoelastic fluid past a deformable surface in the low Reynolds number limit," Physics of Fluids, vol. 19, no. 10, Article ID 104103, 2007.

[24] V. Kumaran, G. H. Fredrickson, and P. Pincus, "Flow induced instability of the interface between a fluid and a gel at low
Reynolds number," Journal de Physique II, vol. 4, no. 6, pp. 893-911, 1994.

[25] A. Silberberg, "Physico-chemical hydrodynamics in turbulent flows close to an interface," Physicochemical Hydrodynamics, vol. 9, no. 3-4, pp. 419-426, 1987.

[26] M. Ulbricht, S. Ozdemir, and C. Geismann, "Functionalized track-etched membranes as versatile tool to investigate stimuliresponsive polymers for "smart" nano- and microsystems," Desalination, vol. 199, no. 1-3, pp. 150-152, 2006.

[27] S. J. Lue, J.-J. Hsu, C.-H. Chen, and B.-C. Chen, "Thermally on-off switching membranes of poly(N-isopropylacrylamide) immobilized in track-etched polycarbonate films," Journal of Membrane Science, vol. 301, no. 1-2, pp. 142-150, 2007. 

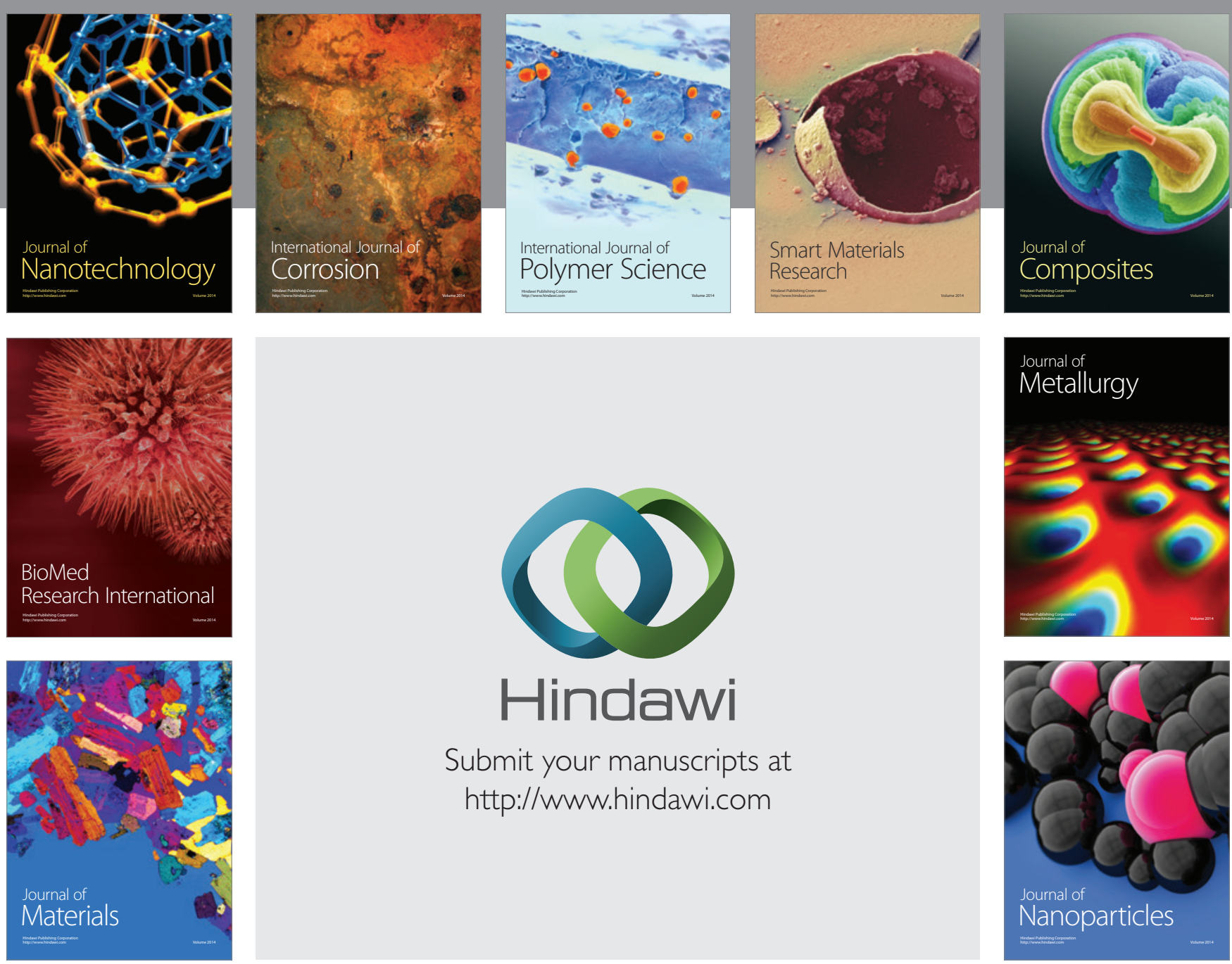

Submit your manuscripts at http://www.hindawi.com
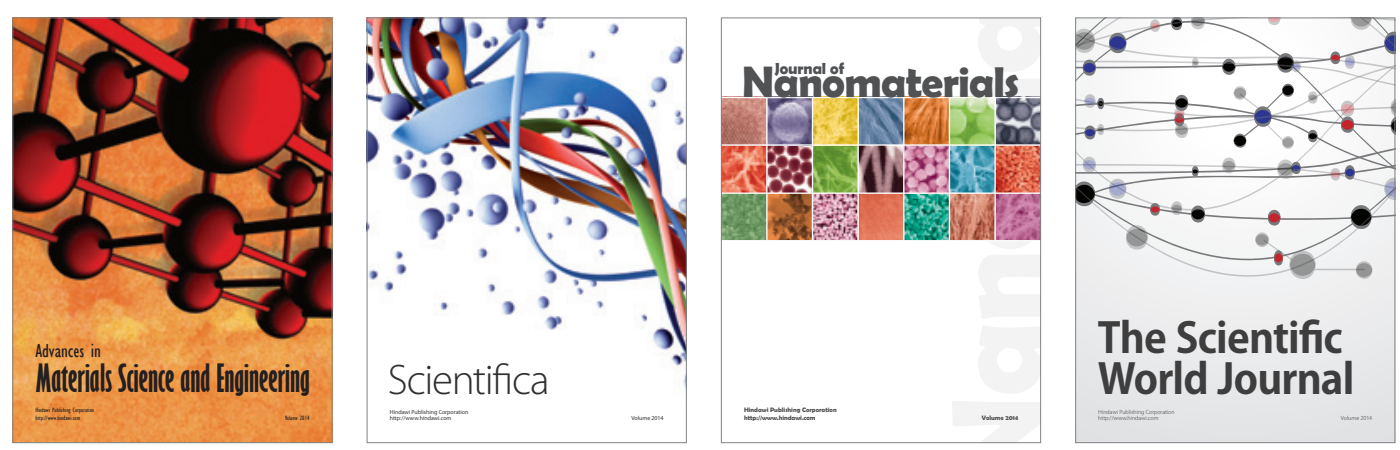

\section{The Scientific World Journal}
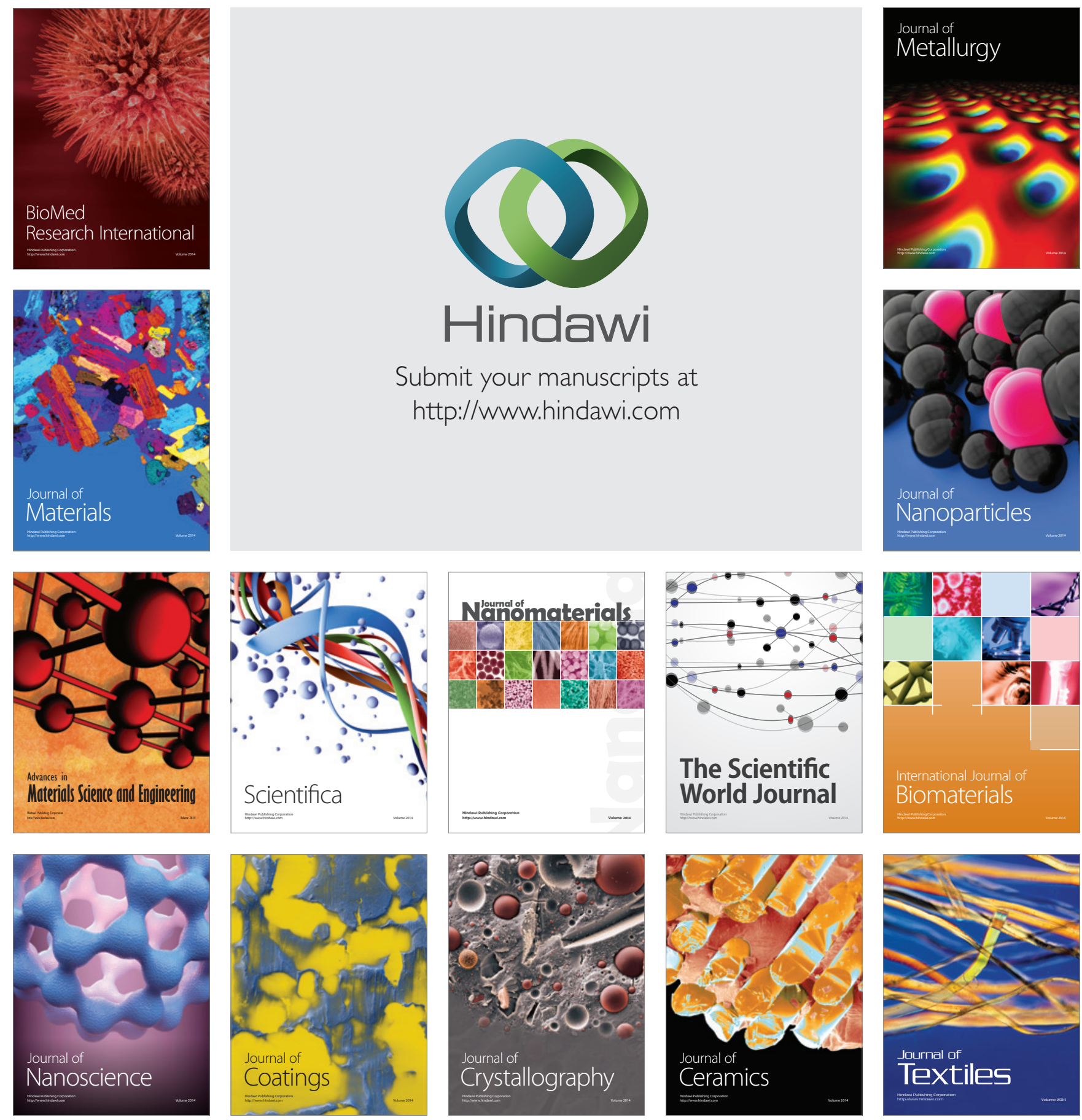